\title{
An Application of Queue management Systems for Congestion Control in Revered Places of Uttarakhand India
}

\author{
Jagdish C. Purohit ${ }^{1}$, O. K. Belwal ${ }^{2}$ \\ ${ }^{1}$ Research Scholar, Department of Statistics, H.N.B. Garhwal University, Srinagar Garhwal \\ ${ }^{2}$ Professor and HOD, Department of Statistics, H.N.B. Garhwal University, Srinagar Garhwal
}

\begin{abstract}
Owing the stressful, busy and fast lifestyle of people in today's highly dynamic circumstances, everybody has a need to get away from it. In this respect going for short and long trips for varied purposes has become an essential part of the life of people. As a result the number of people opting out for travelling to far away tourist destinations is on the rise. Acknowledging the need of traveling of the people in different revered places to find serenity and relax, our proposed approach to the single intersection optimization problem is gradient based, and we derive simulation-based gradient estimators that are more efficient than brute-force finite differences. Here we focus on the single channel waiting line systems with Poisson arrival and exponential service time in revered places. Mathematical models of queuing theory present an interest in modeling, designing and analyzing information network and describe the base for waiting phenomena, services processes, and prioritization of services among others. The other aspect of the study also demands that the expended network in revered places must bring modern technologies along, to serve venerator across the globe for better turnout and efficiency in carrying activities.
\end{abstract}

Keywords: Waiting line system. Congestion, Control, Venerator flow, Queuing theory, Gradient estimators, Simulation

\section{Introduction}

We proposed approach to the single intersection optimization problem is gradient based, and we derive simulation-based gradient estimators that are more efficient than brute-force finite differences furthermore, they can be implemented online, which also differentiates the algorithm from that of Spall and Chin [1].Due to the difficulty of the problem, we apply an approach called smoothed perturbation analysis (SPA), introduced by Gong and Ho [2]. Another simpler technique called infinitesimal perturbation analysis (IPA) is not applicable in the setting [3]. IPA is not applicable, because the sample performance measure is discontinuous in the parameter space. Because of this discontinuity, SPA, which uses conditional expectation, is required. For the single intersection of two one-way streets, we use the framework of $\mathrm{Fu}$ and $\mathrm{Hu}$ [4] to derive unbiased two line gradient estimators for the queue lengths at each of the streets. We then employ these gradient estimators in a stochastic approximation algorithm to optimize the signal light timings. Numerical comparisons with optimization using finite difference estimators illustrate the promise of the proposed approach.

\section{Problem Setting}

The system of interest consists of two one-way streets line 1 line 2 in Figure 1 intersecting at a signal light that has two states:

$\mathrm{A}_{1}$ the light is green for street 1 . This state allows both departures and arrivals at street 1 , but only arrivals at street 2.
$\mathrm{A}_{2}$ the light is green for street 2. This state allows both departures and arrivals at street 2 , but only arrivals at street 1.

The length of a green cycle in states $A 1$ andA2 is denoted by $T_{1}$ and $T_{2}$, respectively. When the light is green for one street, the light is red for the other street. A complete signal cycle is denoted by a green-red sequence, and the time to complete such a cycle is denoted by $T=T_{1}+T_{2}$. We assume that the green-red cycle repeats identically and indefinitely, and without loss of generality assume that the sequence begins with a green for street 1 . In state $\mathrm{A}_{\mathrm{j}}, \mathrm{j}=1.2$, venerator in street $j$ 's queue are served one at a time, according to i.i.d. service times"with mean $1 / \mu_{\mathrm{j}}$, c.d.f. $\mathrm{F}_{j}$ and p.d.f. $\mathrm{F}_{\mathrm{j}}$, whereas in the other queue no venerator are served. All service time and interarrival time distributions are continuous.

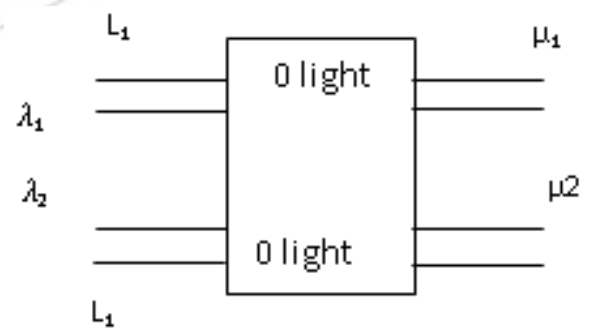

Figure 1: Isolated intersection revert place system visual depiction

If a venerator does not make it through the intersection during a cycle, it must start over with a fresh service time during the subsequent green cycle, i.e., the departure process must start over from scratch once it is realized that the venerator will not exit the queue during the current cycle. Arrivals to each street follow a renewal process with interarrival c.d.f. $G j$, assumed to have finite rate $\lambda_{j}$. Unlike the departure process, the arrival processes to both 


\section{International Journal of Science and Research (IJSR) \\ ISSN (Online): 2319-7064}

Index Copernicus Value (2013): 6.14 | Impact Factor (2015): 6.391

intersection are on in both states. The performance measure of interest is the average number of venerator waiting at revert place for a particular street. By Little's Law, this is essentially equivalent to the average waiting time. We define:

$$
\begin{gathered}
L_{j}(t)=\neq \text { Venerator waiting on street } j \text { at time } t(j \\
=1,2)
\end{gathered}
$$

$\bar{L}_{j}(t)=$ average queue lenght for street $j$ up to time $t$

$$
\begin{gathered}
=\frac{1}{t} \int_{0}^{t} L_{j}(x) d x \\
N=\neq \text { red }- \text { green cycle simulated } \\
\bar{L}=\bar{L}_{1}(N T)+\bar{L}_{2}(N T)
\end{gathered}
$$

In other words, the average total queue length performance measure $\bar{L}$ is taken over N green-red cycles. Note that queue length throughout includes all venerator waiting at the street, even the one currently in service.

The optimization problem is then given by

$$
\min _{T_{1} T_{2}} E[\bar{L}] 1.1
$$

Subject to $T_{1}+T_{2}=T$

Which we propose to solve by satisfying the first-order condition

$$
\nabla_{\theta} E[\bar{L}]=01.2
$$

Where $\theta$ is the vector of controllable variables (parameters), between $T_{1}$ and $T_{2} . T$ is fixed therefore, we can remove the dependence on $\mathrm{T}_{2}$. The optimization problem described by equation 1.1 can be rewritten as

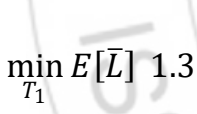

$\theta=$ controllable parameter,

$\dot{\varepsilon}=$ random sequence of numbers defined on a probability space $(\Omega F ; P)$,

We have that a stochastic DES can be represented by the pair $(\theta ; \varepsilon)$, Then the performance measure of interest $L(\theta ; \varepsilon)$ is a random variable on $(\Omega, \mathrm{F}, \mathrm{P})$.

Next, let $F t$ be an increasing family of $\sigma$ algebras on $(' \Omega, F$; $\mathrm{P})$, that is generated by the simulation model up to time $t$. Now let $N$ be the number of cycles simulated and let $T$ be the length of a cycle, then it follows that $N T$ is the duration of a simulation run. The characterization, $Z$, is a $F N T$ measurable random vector. $Z$ is a set of data obtainable from the data generated from a simulation such as queue content, services times, interarrival times, etc. The choice of which data makes up the characterization is a problem dependent decision. The SPA estimator then can stated as

$$
\left(\frac{d E\left[\bar{L}_{j}\right]}{d \theta}\right)_{S P A}=\frac{1}{N} \sum_{k=1}^{N} \frac{\delta}{\delta \theta} L\left(\theta_{1} z_{k}\right) 1.6
$$

Where

$$
\frac{\delta}{\delta \theta} L(\theta, z)=\lim _{\Delta \theta \rightarrow 0} \frac{E \Delta L(\theta, \varepsilon)|z|}{\Delta \theta} 1.7
$$

It is the case that the estimator represented in equation (1.6) can be implemented provided

$\frac{\delta}{\delta \theta} L(\theta, z)$ can be calculated from the simulation run generated by $\left(\theta, \varepsilon_{k}\right)$.

Now we consider the conditions that will ensure the consistency of the estimator represented in equation (1.6).

By the strong law of law numbers we have

$$
\begin{gathered}
\left.\lim _{N \rightarrow \infty} \frac{1}{N} \sum_{k=1}^{N} \frac{\delta}{\delta \theta} L\left(\theta_{1} z_{k}\right)=\lim _{N \rightarrow \infty} \frac{1}{N} \sum_{k=1}^{N} \frac{\delta}{\delta \theta} L\left(\theta, z \theta, \varepsilon_{k}\right)\right) \\
=E \frac{\delta}{\delta \theta} L(\theta, z) \\
=E \lim _{\Delta \theta \rightarrow 0} \frac{E[\delta L(\theta, \varepsilon)|z|]}{\Delta \theta} 1.8
\end{gathered}
$$

To find the value of $\theta$ satisfying the equation (1.2), we use gradient-based simulation optimization via a stochastic approximation recursion of the following form:

So the question is under what conditions

$$
\theta_{n+1}=\Pi_{\theta}\left(\theta_{n}-a_{n} \widehat{\nabla} E\left[\bar{L}\left(\theta_{n}\right]\right) 1.4\right.
$$

$$
\begin{gathered}
\frac{\delta}{\delta \theta} L(\theta, \varepsilon)=\lim _{\Delta \theta \rightarrow 0} E \frac{E[\delta L(\theta, \varepsilon)|z|]}{\Delta \theta} \\
=E \lim _{\Delta \theta \rightarrow 0} \frac{E[\delta L(\theta, \varepsilon)|z|]}{\Delta \theta} 1.9
\end{gathered}
$$

The gradient estimate in 1.4 requires estimators for

$$
\frac{d E\left[\bar{L}_{j}\right]}{d \theta} \cdot j=1,2 \ldots . .1 .5
$$

We assume $T$ is given, so the constraint essentially reduces (1.1) to a two-variable optimization problem. With $T$ fixed a positive perturbation in $T_{1}$ results in a negative perturbation in vice versa. Although we take $T_{1}$ and $T_{2}$ as deterministic, a more general formulation could have $T_{1}$ and $T_{2}$, as random variables, with $\theta$ as a parameter in the distribution of $T_{1}$ or $T_{2}$.

\section{Derivation of Estimators}

Now we examine the concept of SPA as given by Gong and Ho [2]. By defining
Equation 1.9 holds. So basically we are left with requiring an interchange of limit and expectation. Conditions for such an interchange involve applying the dominated convergence theorem. Following the framework of $\mathrm{Fu}$ and $\mathrm{Hu}$ [14], the general SPA estimator consists of an infinitesimal perturbation analysis (IPA) term and a conditional term, the latter due to possible critical event order changes, which intuitively are changes in the order of events in a sample path that drastically alter the performance measure of interest. For instance, in our light setting, a perturbation might lead to one less or one more departure in a given green cycle. How to estimate the probability (rate) of such a change and the subsequent expected effect on the performance measure is the key to deriving the SPA estimator. 


\section{International Journal of Science and Research (IJSR) \\ ISSN (Online): 2319-7064}

Index Copernicus Value (2013): 6.14 | Impact Factor (2015): 6.391

The general form of the SPA estimator is

$$
\left(\frac{d E\left|\bar{L}_{1}\right|}{d \theta}\right)_{S P A}=\frac{d \bar{L}_{j}}{d \theta}+\lim _{\Delta \theta \rightarrow 0} \delta E_{z}\left[\bar{L}_{j}(\beta(\Delta \theta)] 1.10\right.
$$

$\beta(\Delta \theta)$ denotes a critical event change due to a perturbation of $\Delta \theta$ and $\delta E_{z}\left[\bar{L}_{j}(\beta(\Delta \theta)]\right.$ denotesthe corresponding expected change in the performance measure $E_{z}\left[\bar{L}_{j}\right]$. The subscript zdenotes a conditioning on the characterization, which is the set of conditioning quantities on the sample path on which the conditional contribution is estimated, and it will differ for each of the four estimators we derive. In addition to choosingz, the chief difficulty in implementing an SPA estimator is the estimation of the expected change, $\lim _{\Delta \theta \rightarrow 0} \delta E_{z}\left[\bar{L}_{j}(\beta(\Delta \theta)]\right.$ ideally, this quantity would be able to be estimated from the original sample path, which we call the nominal path (NP), but its general form is given as $\lim _{\Delta \theta \rightarrow 0} \delta E_{z}\left[\bar{L}_{j}(\beta(\Delta \theta)]=E_{\mathrm{z}}\left[\bar{L}_{j}^{P P_{1}}-\bar{L}_{j}^{D N P_{1}}\right]\right.$ which is defined by three other sample paths.

NP: nominal path, the original sample path;

PP: perturbed path, limiting version of nominal path on which the critical event change occurs, that is a version of the NP on which the parameter that causes the event change is just big enough to cause the event change;

DNP: degenerate nominal path, limiting version of the nominal path on which no critical event change occurs, that is a version of the NP on which the parameter that causes the event change is just small enough to not cause the event change,

Where the superscripts denote the performance measures on the corresponding sample paths. Over $N$ cycles, the estimator (2.10) becomes

$\left(\frac{d E\left|\bar{L}_{1}\right|}{d \theta}\right)_{S P A}=$

$\frac{d \bar{L}_{j}}{d \theta}+\sum_{i=0}^{N} \lim _{\Delta \theta \rightarrow 0} \frac{P_{z i}\left(\beta_{1}(\Delta \theta)\right)}{\Delta \theta} \lim _{\Delta \theta \rightarrow 0} E_{z i}\left[\bar{L}_{j}^{P P_{i}}-\bar{L}_{j}^{D N P_{i}}\right]$

1.11

Since the optimization is with respect to $T 1$, we will take $\theta=$ $T 1$ throughout. We derive two estimators: Line 1 and Line 2 estimators for each of the two streets, with $l$ and $r$ subscripts denoting line 1 and line2 estimators, respectively. The critical event changes, $\beta(\Delta \theta)$ are quite intuitive: a shortening of a green cycle could cause a departure to be lost during the cycle, whereas a lengthening could allow an additional departure.

\section{Queue 1}

We first consider queue 1 with $\Delta \theta>0$, corresponding to the Line 1 estimator for $\frac{d E\left|\bar{L}_{1}\right|}{d \theta}$, in this case $(\Delta \theta=\Delta \mathrm{T} 1>0)$, there is a positive perturbation in the green signal length of street 1 while keeping the total signal cycle length, $T$, unchanged. Since small perturbations at the end of $T 1$ do not affect the departure.

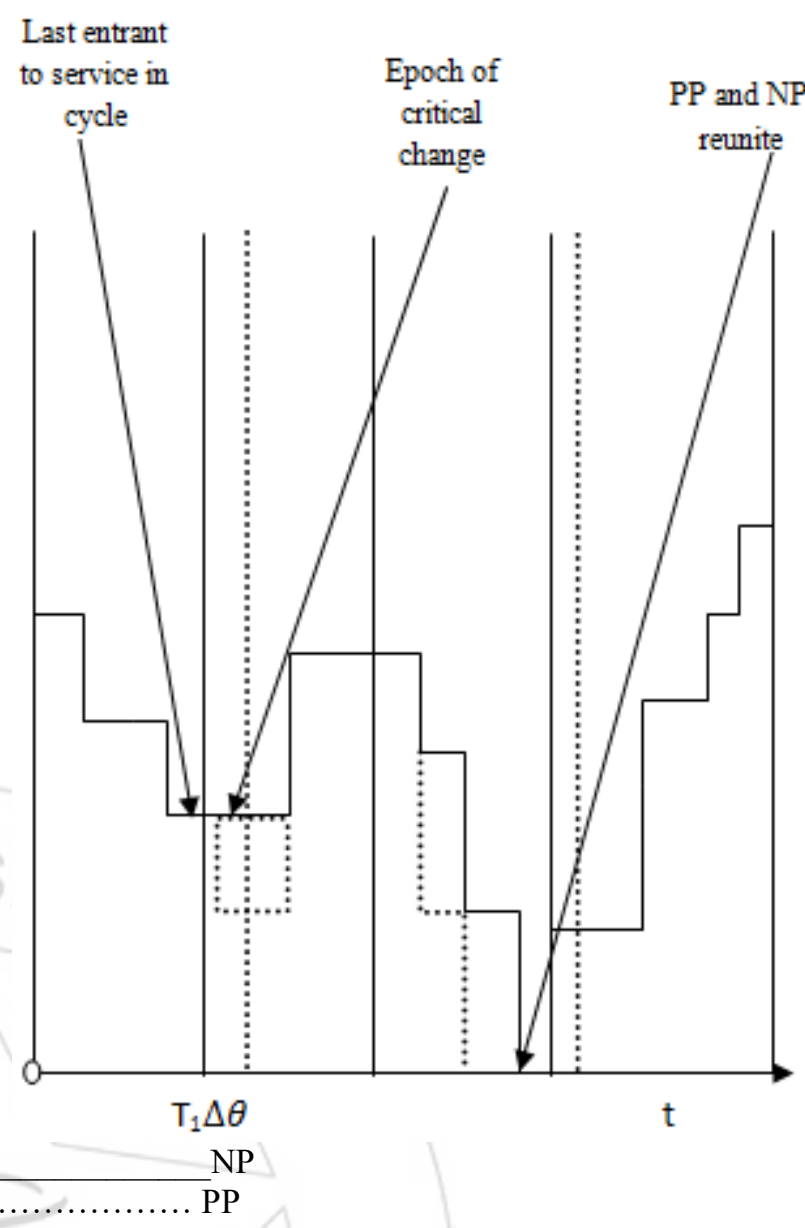

Figure 2: Example of $L_{1}(t)$ sample path for a single intersection optimization system with positive perturbation of $(\Delta \theta>0) T 1$ cycle.

Times of venerator from street 1 , the IPA contribution is zero. A small enough increase in the green signal length would not cause any change in the queue length for street 1 ; however, a large enough increase would lead to an additional departure; in other words, the performance measure is piecewise constant. The critical change in this case is this additional departure. An additional departure is possible if and only if the queue is nonempty at the light change. When nonempty, the last venerator to enter for darsan is the only candidate for a critical change because the probability of more than one critical change is of higher order and thus can be ignored. Thus, we only consider the last venerator as a possible extra departure. To calculate the probability rate and expected effect of this critical change, we condition on all arrivals and darsan times except for the last entry to service during the current $A 1$ state. Since the only critical event change in a cycle is a function of the last entry to darsan, we can index by cycles, and we define:

$\alpha_{\mathrm{i}}=$ time until light change from last entry of service during $i^{\text {th }}$ cycle.

$S=$ set of all darsan times

$S_{1}^{*}=$ last darsan time of $i^{\text {th }}$ cycle.

$A=$ set of all arrival times

$\mathrm{zi}=\frac{S}{\left\{S_{1}^{*}\right\}} \cup A$

where the darsan time for the last venerator to enter darsan (and not depart) in cycle $i$ is greater than $\alpha_{i .}$. DNP and PP are then defined by the critical change occurring precisely at the 


\section{International Journal of Science and Research (IJSR) \\ ISSN (Online): 2319-7064}

Index Copernicus Value (2013): 6.14 | Impact Factor (2015): 6.391

green/red light change, with the darsan times of the last venerator to enter darsan being $\alpha_{i}^{+}$and $\alpha_{i}^{-}$.respectively, where.

$$
\begin{aligned}
& \alpha_{i}^{+}=\alpha+\varepsilon \\
& \alpha_{i}^{-}=\alpha-\varepsilon
\end{aligned}
$$

for $\varepsilon>0$ infinitesimally small. If $X$ denotes a random variable with venerator time distribution $F 1$, then the probability of a critical change is given by

And hence

$$
P\left(\beta_{i}(\Delta \theta)\right)=P\left(X \leq \alpha_{i}+\Delta \theta\right) X \geq \alpha_{i} 1.12
$$

$$
\lim _{\Delta \theta \rightarrow 0} \frac{P\left(\beta_{1}(\Delta \theta)\right)}{\Delta \theta}=\frac{f_{1}\left(\alpha_{i}\right)}{1-F_{1}\left(\alpha_{i}\right)} 2.13
$$

Thus, the estimator given by (2.11) becomes

$$
\begin{aligned}
\left(\frac{d E\left|\bar{L}_{1}\right|}{d \theta}\right)_{S P A, r}= & \frac{1}{N T}\left(\sum_{i=1}^{N} \frac{f_{1}\left(\alpha_{i}\right)}{1-F_{1}\left(\alpha_{i}\right)}\right) E_{z i}\left[\bar{L}_{1}^{P P_{1}}\right. \\
& \left.-\bar{L}_{1}^{D N P_{1}}\right] 1.14
\end{aligned}
$$

We note that $N T$ is the length of each simulation run. To calculate the resulting expected effect, $E_{z}\left[\bar{L}_{1}^{P P_{1}}-\bar{L}_{1}^{D N P_{1}}\right]$ we observe that starting at the critical change, $\bar{L}_{1}^{D N P_{1}}(t)$ will be identical to $L_{1}(t)$, whereas $\bar{L}_{1}^{P P_{1}}(t)$ will be one lower than $L 1(t)$ until $L 1(t)$ empties. Thus, we have that $\bar{L}_{1}^{D N P_{1}}(t)$ $=\bar{L}_{1}^{P P_{1}}(t)+1$ for all $t$ from the epoch of the first light change after the critical change to the time when the system first empties after the critical change (see Figure 2 for an example). Figure 2 shows one possible sample path, we note that the last entrant to darsan that does not exit the system could have also been an arrival to an empty queue. Thus, $E_{z}\left[\bar{L}_{1}^{P P_{1}}-\bar{L}_{1}^{D N P_{1}}\right]=-E\left[\min \left(N T\right.\right.$, inf $\left.\left.\left\{t>\frac{\tau}{L_{1}(t)}=0\right\}\right)\right]-$ $\tau 1.15$

where $\tau=i T-T_{2}$ corresponds to the epoch of the $i^{\text {th }}$ light change from green to red (for street 1). We subtract $\tau$ because the critical change only effects the sample path after its occurrence. Estimation of (1.15) can be done offline as follows. We define $\gamma_{i}=$ Residual interarrival time at the epoch of the $i^{\text {th }}$ light change.

$R_{N}^{(1)}\left(\gamma_{1}\right)=$ Expected time to empty queue 1 , given $N$ venerator in the queue and an initial interarrival time of $\gamma_{i}$

$Q_{i}=$ number in queue at the epoch of the $i^{t h}$ light change from green to red:

Thus. (2.15) can be rewritten as $R_{Q}^{(1)}\left(\gamma_{1}\right)$ and subsequently the estimator given by (2.14) becomes

$$
\left(\frac{d E\left|\bar{L}_{1}\right|}{d \theta}\right)_{S P A, r}=\frac{1}{N T} \sum_{d=1}^{N} \frac{f_{1}\left(\alpha_{i}\right)}{1-F_{1}\left(\alpha_{i}\right)}\left[-R_{\theta_{i}}^{(1)}\left(\gamma_{i}\right)\right] 1.16
$$

Note that the sign of the estimator will be negative, which makes intuitive sense, because an increase in the green signal length should decrease the average queue length.

\section{Queue 2}

We now consider the case of queue 2 with $(\Delta \theta>0)$ to derive the line 2 estimator for $\frac{d E\left|\bar{L}_{2}\right|}{d \theta}$ An increase in $T 1$ affects the entry to darsan of venerator in queue 2 and hence the departure times of venerator in queue 2 , because it results in a decrease in $T_{2}$, delaying the transition from state $A_{1}$ to $A_{2}$ and leading to an IPA perturbation in the departure times of every venerator in the initiating busy period (IBP) of the cycle. If the queue was empty at the beginning of the green period, we say that the particular cycle has no initiating busy period and hence there will be no IPA contribution for that cycle. Also any venerator that arrives after an idle period will not be affected by a perturbation in $T_{1}$, i.e., once the system empties, the perturbation is lost. The critical change for this case is a loss of a departure. A departure by a venerator that is in the initiating busy period may be eliminated by the perturbation and hence represents a potential critical change. To calculate the probability rate and expected effect of each of these possible critical changes, we condition on all arrival times and all darsan times except that of the $k$ th initiating busy period departure of the $i$ th period. We define:

$\alpha_{i}^{k}=$ time until light change from the entry to service of the $k$ th IBP departure;

$S_{1}^{* k}=$ set of all service times of $i$ th cycle prior to $k$ th IBP departure

$$
Z_{i}^{k}=\frac{\delta}{\left\{S_{1}^{*}\right\}} \cup A
$$

$H_{i}=$ number of IBP departures during $i$ th cycle.

$\beta_{1}^{k}=$ critical change cause by $k$ th IBP departure during $i$ th cycle.

$P P_{1}^{k}=$ perturbed path caused by $k$ th IBP departure during $i$ th cycle;

$D N P_{1}^{k}=$ degenerate nominal path caused by $k$ th IBP departure during $i$ th cycle:

If $X$ denotes a random variable with service time distribution $F 2$, then the probability rate of a critical change is given by

$\lim _{\Delta \theta \rightarrow 0} \frac{P\left(\beta_{1}^{k}(\Delta \theta)\right)}{\Delta \theta}=\lim _{\Delta \theta \rightarrow 0} \frac{P\left(X \geq \alpha_{i}^{k}-\Delta \theta / x \leq \alpha_{i}^{k}\right)}{\Delta \theta}=\frac{f_{2}\left(\alpha_{i}^{k}\right)}{F_{2}\left(\alpha_{i}^{k}\right)} 1.17$

Thus, the estimator given by (2.10) becomes

$$
\begin{aligned}
\left(\frac{d E\left|\bar{L}_{2}\right|}{d \theta}\right)_{S P A, r}= & \frac{1}{N T}\left(\sum_{i=1}^{N} H_{i}+\sum_{j=1}^{N} \sum_{k=1}^{H_{i}} \frac{f_{2}\left(\alpha_{i}^{k}\right)}{F_{2}\left(\alpha_{i}^{k}\right)}\right) E z_{1}^{k}\left[\bar{L}_{2}^{P P_{1}^{k}}\right. \\
& \left.-\bar{L}_{2}^{D N P_{1}^{k}}\right] 1.18
\end{aligned}
$$

Estimation of the expected difference between $\bar{L}_{2}^{P P_{1}^{k}}$ and $\bar{L}_{2}^{D N P_{1}^{k}}$ is similar to the previous estimator. The difference in these two performance measures is the difference in the time it takes for the two paths to empty, which can be estimated by simulating the expected time to empty the system, given that the initial queue length is equal to the queue length of the PP path at the time if the light change. Because arrivals are not affected by the perturbation 


\section{International Journal of Science and Research (IJSR) \\ ISSN (Online): 2319-7064 \\ Index Copernicus Value (2013): 6.14 | Impact Factor (2015): 6.391}

of $\mathrm{T}_{1}$ we must consider additional arrivals in the expected difference calculation. Defining.

$R_{N}^{(2)}\left(\gamma_{i}\right)=$ expected time to empty queue 2 , given $N$ cars in the queue and an initial interarrival time of $\gamma_{i}$.

$\gamma_{i}^{k}=$ number in queue immediately after the epoch of the $k^{\text {th }}$ IBP departure during the $i^{\text {th }}$ cycle;

$A_{i}^{k}=$ number of arrivals between $k^{\text {th }}$ entry to service and next light change during $i^{\text {th }}$ cycle.

the final estimator becomes

$\left(\frac{d E\left|\bar{L}_{2}\right|}{d \theta}\right)_{S P A, r}=\frac{1}{N T}\left(\sum_{i=1}^{N} H_{i}+\frac{1}{N T} \sum_{j=1}^{N} \sum_{k=1}^{H_{i}} \frac{f_{2}\left(\alpha_{i}^{k}\right)}{F_{2}\left(\alpha_{i}^{k}\right)} R_{\gamma_{1}^{k}}^{2}+\right.$ $A 1 k(\gamma i) 1.19$

The sign of the estimator will be positive, which makes intuitive sense, because a decrease in the green signal length should increase the average queue length.

\section{Special Cases}

For the special case of exponential interarrival and service times, (1.16), (1.19) respectively simplify to.

$$
\begin{aligned}
& \left(\frac{d E\left[\bar{L}_{1}\right]}{d \theta}\right)_{S P A, r}=-\frac{\mu_{1}}{N T} \sum_{i=1}^{N} R Q_{1}^{(1)} \cdot 1.20 \\
& \left(\frac{d E\left|\bar{L}_{2}\right|}{d \theta}\right)_{S P A, r}=\frac{1}{N T} \sum_{i=1}^{N} H_{i}+\frac{\mu_{2}}{N T} \sum_{i=1}^{N} \sum_{k=1}^{v_{i}} \frac{R^{(1)} \gamma_{1}^{k}+A_{1}^{k}}{e^{\mu_{2} \alpha_{1}^{k}}} 1.21
\end{aligned}
$$

Where the dependence of $R^{(j)} n$ on the residual interarrival time has been removed due to the memoryless property of exponential distribution.

\section{Unbiasedness of the Estimators}

The estimators derived in the previous sections are unbiased if

$E\left[\left(\frac{d E\left[\bar{L}_{j}\right]}{d \theta}\right)_{S P A}\right]=\frac{d E\left[\bar{L}_{j}\right]}{d \theta}, j=1,21.22$

To establish equation (2.22). some additional conditions are required.

(A1) F1(.) is Lipschitz continuous with Lipschitz constant $\mathrm{K} 1$.

(A2) F2(.) is Lipschitz continuous with Lipschitz constant $K 2$.

where for $I \subseteq R$ a function $f: I \rightarrow R$ is said to Lipschitz continuous if there exist a constant $K$ such that $\mid f(x)-$ $f(y)<k x-y$ for all $x ; y I$ and the smallest such $K$ for which this holds is called the Lipschitz constant.

We then have the following result.

\section{Proposition 1}

(i) Under condition (A1), (1.14) is an unbiased estimator for $\frac{d E\left|\bar{L}_{1}\right|}{d \theta}$

(iii) Under condition (A2), (1.18) is an unbiased estimator for $\frac{d E\left|\bar{L}_{2}\right|}{d \theta}$
We establish (i) of proposition 1. The proofs of (2) proceed similarly. Thus their details are omitted here. To proceed, we introduce the following

$$
\begin{gathered}
(\mathrm{NT})=\left\{\mathrm{i} \geq N T: L_{1}(i T, \theta)<0\right\} \\
\mathrm{Ak}=\left\{\mathrm{L} 1(\mathrm{t}, \theta)=L_{1}(t i \theta t \Delta \theta), t=T, 2 T, \ldots . K T\right\} \\
\mathrm{BK}=\left\{L_{1}(t, \theta)=L_{1}(t i \theta t \Delta \theta), t=T, 2 T, \ldots .(K-1) T\right\} \\
V\left\{L_{1}(K T, \theta)=L_{1}(K T, \theta+\Delta \theta)\right\}
\end{gathered}
$$

$\mathrm{K}=1,2 \ldots \mathrm{N}$, where $\Delta \theta=\Delta T_{1}<0$, The set $\mathrm{Z}_{\mathrm{k}}$ is the characterization for our estimator, it contains everything except the darsan time of the last entrant to darsan in period $\mathrm{K}, \mathrm{A}_{\mathrm{k}} \& \mathrm{~B}_{\mathrm{k}}$ are both function of $\Delta \theta$, though we omit the explicit display of the argument, the event $\mathrm{B}_{\mathrm{k}}$ indicates that a perturbation in the vaue of $\theta$ to $\theta+\Delta \theta$ first causes a change in the queue length in period $\mathrm{K}$, the event $\mathrm{AN}$ represents the case where the perturbation does not cause a change in the queue, length over the entire sample path. Thus, $\mathrm{B}_{1} \ldots \ldots \mathrm{BN}$, AN partition our sample space and we can write.

$$
\begin{aligned}
& \frac{d E\left[I_{1}\right]}{d \theta} \\
& =\lim \Delta \theta \\
& -0^{0}\left\{\frac{\left.E\left[I_{1}(\theta+\Delta \theta)-I_{1}(\theta)\right) n\left(A_{n}\right)\right]}{\Delta \theta}\right. \\
& \left.+\frac{\sum_{K=1}^{N} E\left[\left(I_{1}(\theta+\Delta \theta)-I_{1}(\theta)\right) 1\left(B_{k}\right)\right]}{\Delta \theta}\right\} 1.23
\end{aligned}
$$

We first prove the following lemma

Lemma 1. Under condition $\left(\mathrm{A}_{2}\right)$

(a) $\left.E\left[I_{1}(\theta+\Delta \theta)-I_{1}(\theta)\right) 1\left(A_{n}\right)\right]=\Delta \theta X E\left[\frac{1}{N T} \sum_{i=1}^{N} H 1\right]$

(b) $\frac{\left.E\left[I_{1}(\theta+\Delta \theta)-I_{1}(\theta)\right) 1\left(B_{k}\right)\right]=\Delta}{\Delta \theta}=E\left[\frac{1}{N T} \frac{t_{2}(\alpha k)}{1-F_{2}(\alpha k)} R_{k}{ }^{1}\left(\gamma^{k}\right)\right.$

Proof.

$\left(L_{1}(\theta+\Delta \theta)-L_{1}(\theta)\right) 1\left(A_{N}\right)=-\sum_{i=1}^{N} H i \Delta \theta 1.24$

Which established (a)

We consider $\left.E\left[L_{1}(\theta+\Delta \theta)-L_{1}(\theta)\right) 1(B k)\right]$

$\mathrm{K}=\mathrm{T}, 2 \mathrm{~T},---\mathrm{NT}$

First we rewrite it is

We have $E\left[L_{1}(\theta+\Delta \theta) 1(B K) Z K\right]$

$$
E\left[E\left[\left(L_{2}(\theta+\Delta \theta)-L_{1}(\theta)\right) 1 \frac{B k}{Z k}\right]\right.
$$

$$
\begin{aligned}
=E\left[L_{1}(\theta+\Delta \theta) Z k\right. & , \alpha k(\theta)>S k \\
& >\alpha k(\theta+\Delta \theta)](A k-1) X P(\propto k(\theta) \\
& \left.>\int_{x}^{0}>\propto k(\theta+\Delta \theta)\right)
\end{aligned}
$$

$$
\begin{aligned}
& =E\left[L_{1}(\theta+\Delta \theta) Z k, \alpha k(\theta)\right. \\
& \left.\qquad \int_{k}^{0}>\alpha k(\theta+\Delta \theta)\right](A k \\
& \quad-1) X\left(F_{1}(\propto k(\theta+\Delta \theta))-F_{1}(\propto k(\theta))\right) \\
& \geq K_{1} \Delta \theta E\left[L_{1}(\theta+\Delta \theta) Z k, \alpha K(\theta)>\int_{k}^{0}>\propto k(\theta+\Delta \theta)\right.
\end{aligned}
$$

Where $\propto k(\theta+\Delta \theta)=\alpha k(\theta)+\Delta \theta$ and the last inequality follow from assumption $\left(\mathrm{A}_{1}\right)$ to bound the expectation, we introduce notation for a renewal counting process based on 


\section{International Journal of Science and Research (IJSR) \\ ISSN (Online): 2319-7064 \\ Index Copernicus Value (2013): 6.14 | Impact Factor (2015): 6.391}

the arrivals (without darsan). Let $\left\{\mathrm{X}_{\mathrm{n}}, \mathrm{n}=1,2 ..\right\}$ be a sequence of i.i.d. interarrival times with common distribution $\mathrm{G}_{1}$ and denote the associated counting process $\{P(t) t>0\} G_{n}$ generates non negative interarrival times with a finite rate $(\lambda 1)$ thus $\mathrm{G} 1(0)>1$ noting the arrivals are independent of $\theta$, from renewal theory we have.

$$
\begin{gathered}
E\left[\frac{\text { Sup }}{\Delta \theta} L_{1}(\theta+\Delta \theta) Z K, \alpha k(\theta)>S k^{0}>\alpha k(\theta+\Delta \theta)\right] \\
\geq E[P(N T)]<D
\end{gathered}
$$

So by invoking the dominated convergence theorem (DCT), we have

$$
\begin{aligned}
& \frac{\operatorname{Lim}}{\Delta \theta} E \frac{\left[I_{1}(\theta+\Delta \theta) 1(B k)\right]}{\Delta \theta}=E \frac{\lim }{\Delta \theta} E \frac{\left(L_{1}(\theta+\Delta \theta) 1(B k) z k\right]}{\Delta \theta} \\
& E \frac{\lim }{\Delta \theta s o} \frac{\left((\alpha k(\theta+\Delta \theta))-F_{1}(\alpha k(\theta))\right]}{\Delta \theta} X \frac{\lim }{\Delta \theta s o} E\left[L_{1}(\theta\right. \\
+\Delta \theta) Z k, \alpha k(\theta)>S k^{0} & >\alpha k(\theta+\Delta \theta)] X I(A k-1)] \\
= & E\left[t_{1}(\propto k) E\left[L^{-P P k}(t)\right]\right] \\
= & E\left[t_{2}(\propto k) E\left[L^{-P P k}(t)\right]\right] \\
= & \left.E \frac{t_{1}(\propto k)}{1-F_{1}(\propto k)}\left\{S k^{\infty}<\propto k(\theta)\right\} E\left[L_{1}^{-P P k}(t)\right]\right]
\end{aligned}
$$

We can similarly show

$$
\begin{aligned}
& \frac{\lim }{\Delta \theta s o} \frac{E\left[L_{1}(\theta) 1(B k)\right.}{\Delta \theta} \\
= & E\left[\frac { f _ { 1 } ( \alpha k ) } { 1 - f _ { 1 } ( \alpha k ) } \left\{S k^{0}\right.\right. \\
< & \left.\alpha k(\theta)\} E\left[L_{1}{ }^{D N P k}(t)\right]\right]
\end{aligned}
$$

\section{Numerical Results}

The first case $\left(\mathrm{V}_{1}\right)$ corresponds to symmetric street flows and signal timings: $\mu_{1}=\mu_{2}=2.0, \quad \lambda_{1}=\lambda_{2}=4.5 \quad \mathrm{~T}=30$, $\mathrm{T}_{1}=\mathrm{T}_{2}=15$, the second case $\left(\mathrm{V}_{2}\right)$ is an asymmetric system $\mu_{1}=1.5 \mu_{2}=.75, \lambda_{1}=\lambda_{2}=5.0 \mathrm{~T}=50, \mathrm{~T}_{1}=20, \mathrm{~T}_{2}=35$ the simulation were carried out $\mathrm{N}=5,000$ cycles over 5000 replications

Table 1: SPA gradient estimate simulation result for the isolated intersection over all pulse setting for $\left(\mathrm{V}_{1}\right)$ (standard error parenthesis)

\begin{tabular}{|c|l|c|}
\hline Estimator & $\frac{d E\left[\bar{L}_{1}\right]}{d \theta}($ std. err $)$ & $\frac{d E\left[\bar{L}_{2}\right]}{d \theta}($ std.err $)$ \\
\hline SPA $(\lim 1)$ & $-2.324(.010)$ & $2.3765(0.0001)$ \\
\hline FD $(.05)$ & $-2.2403(.042)$ & $2.7834(0.0672)$ \\
\hline
\end{tabular}

Table 2: SPA gradient estimate simulation results for $V_{2}$ (standard error in parenthesis)

\begin{tabular}{|c|c|c|}
\hline Estimator & $\frac{d E\left[\bar{L}_{1}\right]}{d \theta}($ std.err $)$ & $\frac{d E\left[\bar{L}_{2}\right]}{d \theta}($ std.err $)$ \\
\hline SPA $(\lim 2)$ & $-6.203(.0043)$ & $0.00675(.0008)$ \\
\hline FD $(.05)$ & $-5.531(0.3215)$ & $0.08932(0.0023)$ \\
\hline
\end{tabular}

The results are shown in table $1 \& 2$ the FD estimates are quit sensitive to the difference value chosen, the best results are reported here, where, the number in parentheses following the heading, ,FD ${ }^{\text {ee }}$ in the tables indicates the specific difference value, even so, that SPA estimator is more precise, with a standard error always at least an order of magnitude vector and it also more stable and computationally efficient, in fact, when I is also desired the FD estimators require (on average) nearly three times as much computation time, the confidence intervals for all estimators overlap for these cases.

\section{Conclusions}

As far as we are aware, this is the first successful attempt to apply direct stochastic gradient estimation techniques to a venerator queue in revered places for optimization setting. This algorithm has also been converted to computer programming. The resulting estimators demonstrated superior computational performance over FD estimators, and in addition can be used on line with real-time venerator updating systems, because unlike FD estimators, they do not require altering the parameter values. Thus, although we have considered only a single intersection, this work constitutes an important stepping stone in the foundation of simulation-based venerator queue management.

\section{References}

[1] J.C. Spall and D.C. Chin, Traffic-Responsive Signal Timing for System-Wide Traffic Control, "Transportation Research - C, Vol.5, pp.153-163, 1997.

[2] W.B. Gong and Y.-C. Ho, Smoothed Perturbation Analysis of Discrete-Event Dynamical Systems, "IEEE Transactions on Automatic Control, vol.32, pp.858867,1987

[3] L. Head, F.W. Ciarallo, D. Lucas, and V. Kaduwela, A Perturbation Analysis Approach to Traffic Signal Optimization, "INFORMS National Meeting, Washington, D.C., May 5-8, 1996.

[4] M.C. Fu and J.Q. Hu, Conditional Monte Carlo: Gradient Estimation and Optimization Applications, Kluwer Academic Publishers, Boston, MA; 1997.

[5] Y.-C. Ho and X.-R. Cao, Perturbation Analysis and Optimization of QueueingNetworks, “J. Optim. Theory Appl. Vol.40, pp.700-714, 1983.

[6] Y.-C. Ho, Perturbation Analysis: Concepts and Algorithms, "Proceedings ofthe Winter Simulation Conference, pp.231-240, 1992.

[7] K.N. Hewage and J.Y. Ruwanpura, Optimization of Traffic Signal LightTiming Using Simulation, "Proceedings of the Winter Simulation Conference, pp.1428-1433, 2004.

[8] G. Improta and G.E. Cantarella, Control Systems Design for an Individual Signalised Junction, "Transportation Research B, Vol.18, pp.147-167, 1984.

[9] A.M. Law and W.D. Kelton, Simulation Modeling and Analysis (3rd Edition), McGraw-Hill, Boston, MA; 2000.

[10]D.C. Lee, Applying Perturbation Analysis to Traffic Shapping, "Computer Communications, Vol.24, pp.798810, 2002.

[11]R.V. Lindgren and S. Tantiyanugulchai, Microscopic Simulation of Traffic ata Surburban Interchange, "Institute of Transprtation Engineers 2003 Annual Meeting, 2003.130 


\section{International Journal of Science and Research (IJSR) \\ ISSN (Online): 2319-7064 \\ Index Copernicus Value (2013): 6.14 | Impact Factor (2015): 6.391}

[12] J.D.C. Little, The Synchronisation of Traffic Signals by Mixed -integer-linear-programming, "Operations Research, Vol.14, pp.568-594, 1966.

[13]J.D.C. Little, M.D. Kelson, and N.H. Gartner, MAXBAND: A Program forSetting Signals on Arteries and Triangular Networks, "Transportation Research Record, No.795, pp.40-46, 1981.

[14]F.L. Mannering, W.P. Kilareski, and S.S. Washburn, Principles of Highway Engineering and Traffic Analysis, John Wiley, Hoboken, NJ; 2005.

[15]A. J.Miller, A Computer Control System for Tra $\pm \mathrm{c}$ Networks, "Proc. $2^{\text {nd }}$ Int. Symp. Traffic Theory, pp.200220, 1963.

[16]M. Papageorgiou, C. Diakaki, V. Dinopolou, A. Kotsialos, and Y. Wang, Review of Road Traffic Control Strategies, "Proceeding of the IEEE, Vol.91, pp.2043-2067, 2003.

[17] D.I.Robertson, TRANSYT Method for Area Traffic Control", Traffic Engineer-ing\& Control, Vol.10, pp.276-281, 1969.

[18] P.B. Hunt, D.L. Robertson, and R.D. Bretherton, The SCOOT On-line Traffic Signal Optimization Technique, "Traffic Engineering \& Control, Vol.23, pp.190-192, 1982.

[19] R.P. Roess, E.S. Prassas, and W.R. McShane, Traffic Engineering, Prentice Hall, Upper Saddle River, NJ; 2004.131

[20] S. Sen and L. Head, Controlled Optimization of Phases at an Intersection, "Transportation Science, Vol.31, pp.5-17, 1997.

[21] S. Algers et. al. SMARTEST: Review of MicroSimulation Models, "1997.

[22]G. Sun, C.G.Cassandras, Y. Wardi and C.G. Panayiotou, Perturbation Analysis of Stochastic Flow Networks, "Proceedings of the 42nd Conference Decisionand Control, 2003.

[23]J.C. Spall, Multivariate Stochastic Approximation Using Simultaneous Per-turbation Gradient Approximation, "IEEE Transactions on Automatic Control, Vol.37, pp.332-341, 1992.

[24]C. Stamatiadis and and N.H. Gartner, MULTIBAND96: A Program for Variable Bandwidth Progression Optimization of Multiarterial Traffic Networks, "Transportation Research Record, No.1554, pp.917, 1996.

[25] M.L. Tartaro, C. Toress, and G. Wainer, UDefining Models of Urban Traffic Using the TSC Tool, "Proceedings of the Winter Simulation Conference, pp.1056-1063, 2001.

[26] Y.Wardi, B. Melaned, C. Cassandras, and C. Panayiotou, IPA Gradient Estimators in Single-node Stochastic Fluid Models, "Journal of Optimization Theory and Applications, Vol.115, No.2, pp.369-406, 2002.132

[27]F.V. Webster, Traffic Signal Settings, "Road Research Technical Paper No.39, Research Laboratory, London, UK, 1958.

[28]A. Vogel, C. Goerick and, W. Von Seelen, Evolutionary Algorithms for Optimizing Traffic Signal Operation, "Proceedings of the European Symposium on Intelligent Techniques (ESIT 2000), Aachen, Germany, pp.83-91, Sept 14-15, 2000.
[29] S. Meyn, Sequencing and Routing in Multiclass Networks. Part I: Feedback Regulation, "Proceedings of the IEEE International Symposium on Information Theory, pp.4440-4445, 2000.

[30] G. Sun C.G. Cassandras, Y. Wardi, C.G. Panayiotou, and G. Riley, Perturbation Analysis and Optimization of Stochastic Flow Networks, "IEEE Trans-action on Automatic Control, AC-49, 12, pp.2113-2128, 2004.133

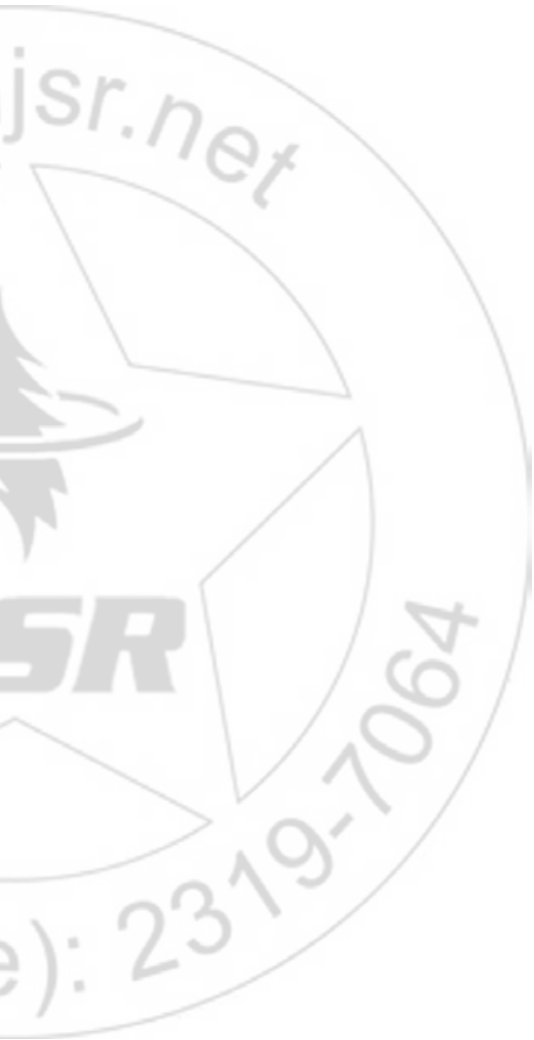

